\title{
Design \& Analysis of Optical Lenses by using 2D Photonic Crystals for Sub-wavelength Focusing
}

\author{
Rajib Ahmed $^{* 1}$ \\ Masters on Photonic Networks \\ Engineering, \\ Scuola Superiore Sant'Anna, \\ Pisa, Italy \\ School of CSE, University of \\ Information Technology \& Science, \\ Dhaka, Bangladesh
}

\author{
Mahidul Haque Prodhan ${ }^{2}$ \\ Dept. of Applied Physics, \\ Electronics \& Communication \\ Engineering, \\ University of Dhaka, \\ Dhaka, Bangladesh
}

\author{
Rifat Ahmmed ${ }^{3}$ \\ Dept. of Electronics \& \\ Telecommunication Engineering, \\ Rajshahi University of Engineering \\ \& Technology (RUET), \\ Rajshahi, Bangladesh
}

\begin{abstract}
D Photonic lenses (Convex-Convex, Convex-Plane, Plane-Convex, Concave-Concave, Concave-plane, and PlaneConcave) have been designed, simulated and optimized for optical communication using FDTD method. The effect of Crystal structures (Rectangular, Hexagonal, Face centered Cubic (FCC), Body centered Cubic (BCC), variation lattice constant $(\Lambda)$, hole radius(r), reflective index $(n)$, is demonstrated to get optimized parameters. Finally, with optimized parameters the effect of variation of lens radius on focal lengths and Electrical Field Intensity (Ey) is analyzed. Like optical lens, the focal length of photonic lens is also increased with lens radii, has dependency on optical axis. Moreover, with optimized parameters, ConcaveConcave lens have been found as an optimal photonic lens that show sub-wavelength focusing with spatial resolutions$9.22439 \mu \mathrm{m}$ (Rectangular crystal), 7.379512 $\mu \mathrm{m}$ (Hexagonal Crystal), 7.840732 $\mu \mathrm{m}$ (FCC, BCC).
\end{abstract}

Keywords-Photonic crystals; photonic lens; body centered cubic; face centered cubic.

\section{INTRODUCTION}

In the recent years, a lot of research has been focused on developing micro and nano photonic devices by using Photonic Crystals (PCs). Photonic Crystals (PCs) are artificial structure in which the periodic variation of dielectric constant is used to control the flow of light. PCs may be 1D, 2D \& 3D crystal structure. Unlike electronic crystals (which are natural structure) that control the flow of electron by periodic variation of electron potential and follow Schrödinger equation, PCs follow Maxwell's equation [1].

Now a day, from the idea of controlling light by means of Photonic Crystal (PC) has led to many proposals and implementations for novel devices including different types of focusing elements and nano-scale imaging field. This is possible only for negative refraction effect. The traditional limitation on Optical lenses is no lens can focus light onto an area smaller than a square wavelength, overcome by Photonic lenses as Super lenses [2].

We have proposed different photonic lenses (ConvexConvex, Convex-Plane, Plane-Convex, Concave-Concave, Concave-plane, Plane-Concave) with Rectangular, Hexagonal, Face-centered Cubic (FCC), Body centered Cubic (BCC) photonic crystals for sub-wavelength focusing and try to optimize the parameters (Hole Radius(r), Positions, Reflective Index (n), Input Signal wavelength $(\lambda)$, lattice constant $(\Lambda)$, Distances or position of lens, Focal length (f), Spatial resolution $(\Delta \mathrm{x})$ ) to get optimal crystal structure (Rectangular) with optimal photonic lens (Concave-Concave). Moreover, focusing of the light is found smaller then square input wavelength. Thus Sub-wavelength focusing has been found for photonic lenses.

The Sub-wavelength focusing has great Effect in nanoscale imaging, coupling large bandwidth waveguide to lower bandwidth waveguide applications and so on.

\section{THEORITICAL BACKGROUND}

Propagation of light in photonic crystals is described by Maxwell's equations. Solving the equation under the condition of no free charges or currents, we get the following equation

$$
\nabla \times\left(\frac{1}{\varepsilon(\mathrm{r})} \nabla \times \mathrm{H}(\mathrm{r})\right)=\left(\frac{\omega}{c}\right)^{2} \mathrm{H}(\mathrm{r})
$$

Equation (1) is known as the master equation [1]. Here, $\varepsilon$ (r), H(r), $\omega$ and c represent the dielectric constant, the magnetic field distribution, the frequency and the speed of light in vacuum, respectively. Eq. (1) follows the BlochFloquet theorem, which proves that waves in $3 \mathrm{D}$ periodic media can propagate without scattering. Their behavior governed by a periodic function multiplied by a plane wave. The Bloch modes have the form

$$
\boldsymbol{H}(\boldsymbol{r})=e^{i(k \cdot r-\omega t)} \boldsymbol{H}_{\boldsymbol{n}, \boldsymbol{k}}
$$

With eigenvalues $\omega_{n}(k)$, where $H_{n, k}$ is a periodic envelope function satisfying:

$$
(\nabla+i \boldsymbol{k}) \times \frac{1}{\varepsilon}(\nabla+i \boldsymbol{k}) \times \boldsymbol{H}_{n, \boldsymbol{k}}=\left(\frac{\omega_{n}(\boldsymbol{k})}{c}\right)^{2} \boldsymbol{H}_{n, \boldsymbol{k}}
$$

yielding a different Hermitian eigen problem over the primitive cell of the lattice at each Bloch wave vector $\mathrm{k}$. This primitive cell is a finite domain if the structure is periodic in all directions, leading to discrete eigenvalue labeled by $n=1$, $2 \ldots$ These eigenvalues when plotted with respect to the wave vector, $\mathrm{k}$ forms the band diagram or dispersion diagrams [4].

These eigenvalues are periodically repeating functions of $\mathrm{k}$. The values at $\mathrm{k}$ are same as the values obtained at $\mathrm{k}+\mathrm{Gj}$. 
Here, $\mathrm{Gj}$ denotes the primitive reciprocal lattice vector [1]. Thus, eigenvalues can be computed for only the wave vector, $\mathrm{k}$. This unit cell repeats to form the entire lattice structure [1].

\section{LAYOUT DESIGN AND SIMULATION}

We take $\mathrm{SiO}_{2}$ as a wafer material (reflective index 1.447) with dimension- length-30 $\mu \mathrm{m}$ and width- $48 \mu \mathrm{m}$, Gaussian modulated continuous wave as an input signal with wavelength $1.55 \mu \mathrm{m}$. Both transverse and injection type of the input is Modal. The input signal (Fig. a) used in the simulation expressed as:

$$
\mathrm{E}_{\mathrm{y}}{ }^{\text {inc }}\left(\mathrm{x}, \mathrm{z}_{\text {inc }}\right)=\mathrm{AT}(\mathrm{t}) \mathrm{F}\left(\mathrm{x}, \mathrm{z}_{\text {inc }}\right) \sin \left(\mathrm{wt}+\theta_{\mathrm{i}}\right)(4)
$$

Where, $\mathrm{A}$ is the field amplitude and $\mathrm{F}\left(\mathrm{x}, \mathrm{Z}_{\text {inc }}\right)$ is the transverse field location at the incident field location $\mathrm{z}_{\text {inc }}$. The initial offset $\theta_{\mathrm{i}}$ is the phase difference between points in the incidence plane. This offset can be adjusted to define the direction of the incident field.
Time [Sec]

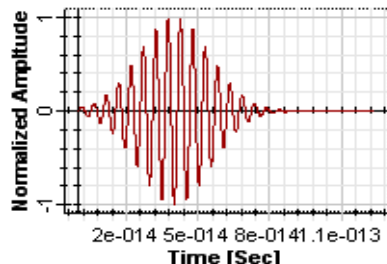

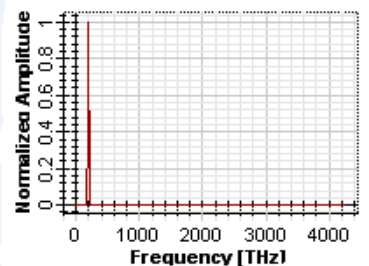

Frequency [THz]
Figure a: Gaussian modulated continuous signal with time and frequency

The propagation of light in the waveguides is simulated by 2D Finite-difference time-domain (FDTD) method using a standard simulator. FDTD is a time-domain numerical method used for modeling the propagation of electromagnetic waves in optical media, which is based on the discretization of Maxwell's equations in differential form in free space. Time domain methods have been found to be very accurate in simulating the propagation dynamics of signals in periodic dielectric media [3]. We simulate for TE mode, actual Mesh used- $0.075 \mu \mathrm{m}$ (delta X) X $0.075 \mu \mathrm{m}$ (delta $Z$ ) with number of mesh cells $400(\mathrm{X})$ and $650(\mathrm{Z})$. For result finalization the simulation has been done for 4000 time steps. The Anisotropic Perfectly Matched Layer (APML) boundary condition is used with 20 layers. Figure1 shows the Simulation setup for Convex-Convex lenses, 2D-light propagation during simulation and finally resulted $3 \mathrm{D}$-Poynting Vector \& DFT $\mathrm{E}_{\mathrm{y}}$ curve. Moreover, Fig. 2 and Fig. 3 show the Simulation setup and 2D focusing for Convex-Plane (A2), Plane-Convex (B2), Concave-Concave (C2), Concave-plane (D2), Plane-Concave (E2) Lenses.

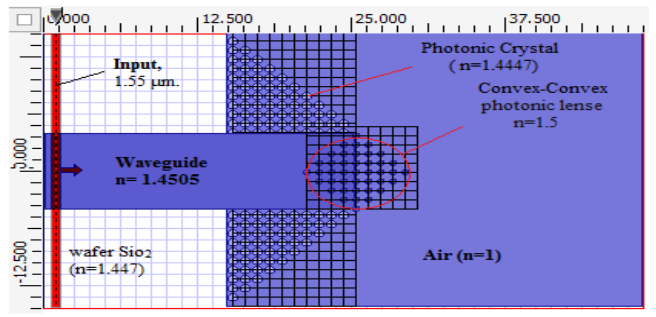

(A1)

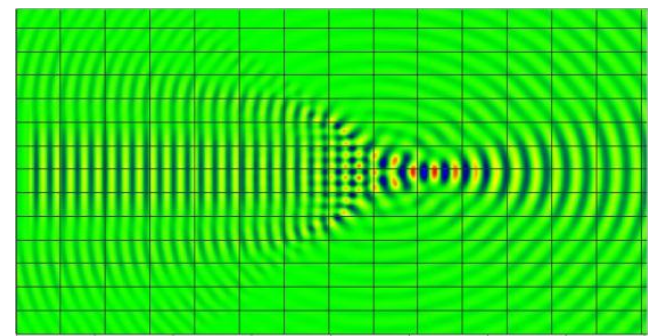

(B1)

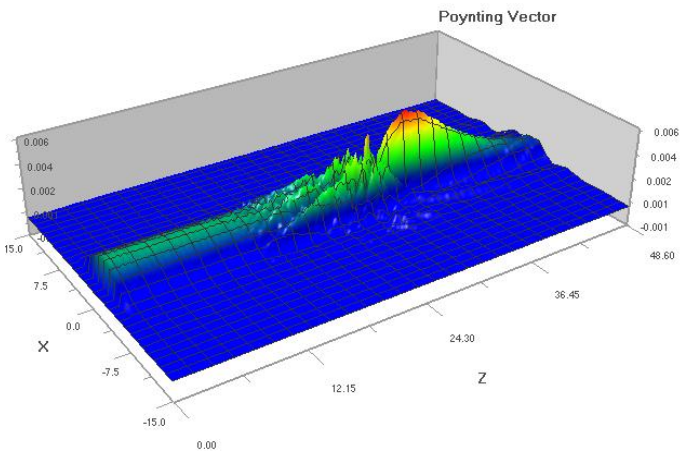

(C1)

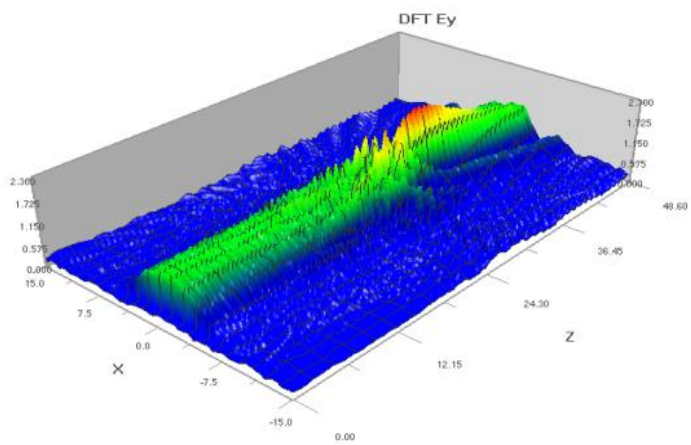

(D1)

Figure 1. (A1) Experimental Setup, (B1) 2D Simulation, (C1) Poynting Vector, (D1) DFT E y

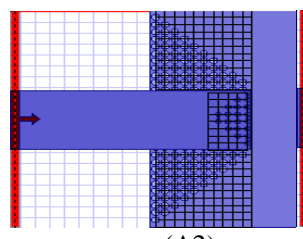

(A2)

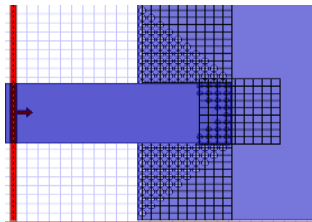

(D2)

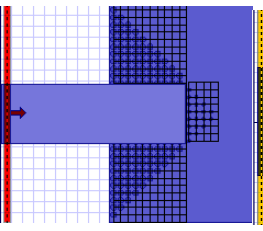

(B2)

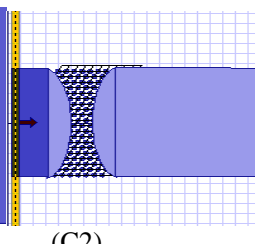

(C2)

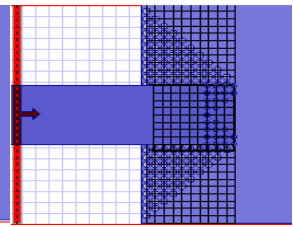

(E2)

Figure 2. Experimental Setup Convex-Plane (A2), Plane-Convex (B2), Concave-Concave (C2), Concave-plane (D2), Plane-Concave (E2) Lenses. 


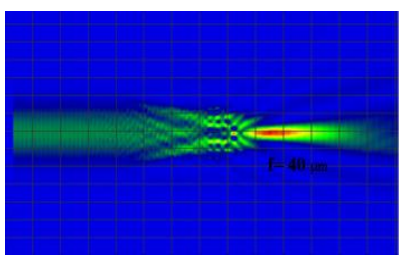

(A3)

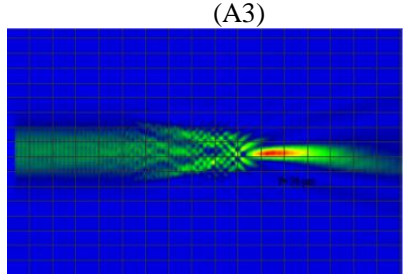

(C3)

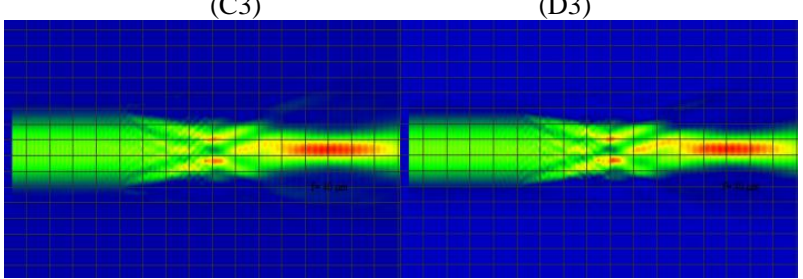

(E3)

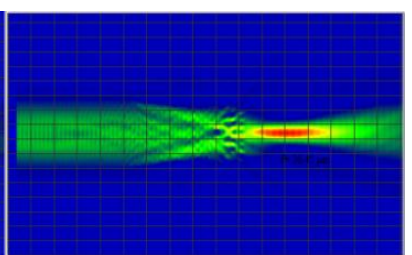

(B3)

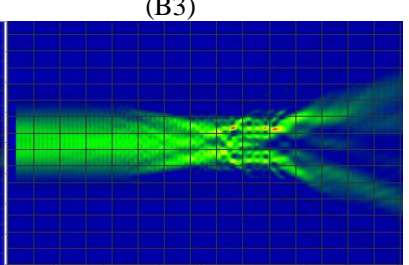

(D3)
Figure 3. 2D focusing for (A3) Convex-Plane, (B3) Plane Convex, (C3) Concave-Concave, (D3) Concave-plane and (E3) Plane-Concave Lenses.

\section{RESULT ANALYSIS}

We have done FDTD simulation for implementation of optical focusing with Rectangular, Hexagonal, Face-centered Cubic (FCC), and Body centered Cubic (BCC) photonic crystals. For Convex-Convex lens (FCC), with hole radius(r) increases, the focal length $(f)$ and the spatial resolution $(\Delta x)$ decrease but DFT $\mathrm{E}_{\mathrm{y}}$ increases. After, hole radius, $\mathrm{r}=0.3 \mu \mathrm{m}$, we get no fixed focal length $\&$ spatial resolution (Table-1). In all case, the lens $\operatorname{radius}(\mathrm{R}=8.2 \mu \mathrm{m}) \&$ lattice constant $(\Lambda=1 \mu \mathrm{m})$ are kept constant.

Similarly, for Convex-Plane, Plane-Convex, ConcaveConcave, Concave-plane, Plane-Concave lenses with Rectangular, Hexagonal, Face-centered Cubic (FCC), Body centered Cubic (BCC) photonic crystals the hole radius, distances or position of lenses from waveguide are varied (Table- 1 to Table-10). The optimal values of distances or position of lenses is zero $\mu \mathrm{m}$, hole radiuses, $\mathrm{r}=0.2 \mu \mathrm{m}$ (FCC), $\mathrm{r}=0.3 \mu \mathrm{m}$ (BCC, Rectangular), $\mathrm{r}=0.4 \mu \mathrm{m}$ (Hexagonal). Moreover, like convention optical lenses, the focal length $(f)$ of photonic lenses also decreases with increase of diameter of lens (Table-11). From the variation of reflective index (n) of the lens, the optimal value is found $n=1.4505$ (Table-12). Finally, the dependence of variation of focal length $(f)$, spatial resolution $(\Delta x)$ on input signal wave length $(\lambda)$ is analyzed to get optimal values, $\lambda=1.55 \mu \mathrm{m}$ (for TM Mode)(Table-14) .

But with TE Mode we get no focal length $(f)$, spatial resolution $(\Delta \mathrm{x})$ before $\lambda=1.35 \mu \mathrm{m}$ (Table-13). With optimized parameters (Hole Radius(r), Positions, Reflective Index(n), Input Signal wavelength $(\lambda)$, lattice $\operatorname{constant}(\Lambda)$ etc. the optimized values of focal lengths $(f): 40 \mu \mathrm{m}, 32 \mu \mathrm{m}, 34 \mu \mathrm{m}, 34 \mu \mathrm{m}$ and spatial resolution $9.22 \mu \mathrm{m}, 7.37 \mu \mathrm{m}, 7.84 \mu \mathrm{m}, 7.84 \mu \mathrm{m}$ (for Rectangular, Hexagonal, BCC, FCC respectively) are found. Thus, the best result is found for Rectangular photonic crystal configuration (Table-15).

Finally, with optimized parameter and photonic crystal structure (rectangular)- DFT $\mathrm{E}_{\mathrm{y}}$ curve (Figure4) for ConvexPlane, Plane-Convex, Concave-Concave, Concave-plane, Plane-Concave Lenses are found. From those DFT $\mathrm{E}_{\mathrm{y}}$ curves, the maximum value and concentrations of DFT Ey across zero position (along X-axis) is occurring for Concave-Concave photonic lens. Moreover, sub-wavelength focusing are occurred for all proposed photonic lenses i.e focusing from the lenses will occur less than the half of the input signal wavelength (Fig. 3). Therefore, the limitation of normal glass lenses can be overcome by photonic lenses. Like convention optical lens, photonic lens also having dependence on optical axis and Concave-Concave photonic lens is the optimal configuration for light focusing (Table-15).

\begin{tabular}{|c|c|c|c|c|c|c|c|c|c|c|c|}
\hline \multirow[t]{3}{*}{\begin{tabular}{|l} 
Radius \\
of lens \\
$\mathrm{R}(\mu \mathrm{m})$
\end{tabular}} & \multirow[t]{3}{*}{$\begin{array}{c}\text { Lattice } \\
\text { constant, } \\
\Lambda(u m)\end{array}$} & \multirow[t]{3}{*}{$\begin{array}{l}\text { Hole } \\
\text { Radius, } \\
\mathrm{r}(\mathrm{um})\end{array}$} & \multirow[t]{3}{*}{$\begin{array}{c}\text { Focal } \\
\text { Length, } \\
f(\mu \mathrm{m})\end{array}$} & \multirow[t]{3}{*}{$\begin{array}{c}\text { Ey(DFT) } \\
\left(\mathrm{xe}^{-0.101}\right) \\
\mu \mathrm{m}\end{array}$} & \multirow{3}{*}{\begin{tabular}{|c|} 
Spatial \\
Resolution, \\
$\Delta x$ \\
$=1.221 *(F / D)$ \\
$\mu \mathrm{m}$ \\
\end{tabular}} & \begin{tabular}{|l|} 
Radius \\
of lens \\
$R(\mu \mathrm{m})$
\end{tabular} & $\begin{array}{c}\text { Lattice } \\
\text { constant, } \\
\Lambda(\mathrm{mm})\end{array}$ & $\begin{array}{c}\text { Hole } \\
\text { Radius, }, \mathrm{r} \\
(\mu \mathrm{m})\end{array}$ & $\begin{array}{l}\text { Focal } \\
\text { Length, } \\
f(\mu \mathrm{m})\end{array}$ & $\begin{array}{c}\text { Ey(DFT) } \\
\left(x e^{20 x}\right) \\
\mu \mathrm{m}\end{array}$ & $\begin{array}{c}\text { Spatial } \\
\text { Resolution, } \\
\Delta x \\
=1.22 \lambda^{*}(F / D) \\
\mu \mathrm{m}\end{array}$ \\
\hline & & & & & & \multirow{10}{*}{8.2} & \multirow{10}{*}{1} & .05 & 38 & 3 & 8.763171 \\
\hline & & & & & & & & .1 & 37.5 & 3.6 & 8.647866 \\
\hline \multirow{8}{*}{8.2} & \multirow{8}{*}{1} & .05 & 38 & 3.3 & 8.763171 & & & .2 & 36.45 & 5.9 & 8.405726 \\
\hline & & .1 & 37.5 & 3.6 & 8.647866 & & & $\frac{3}{4}$ & 33 & 3.2 & 7.610122 \\
\hline & & .15 & 34 & 3.4 & 7.840732 & & & .5 & 30 & 2.4 & $\begin{array}{l}7.3 / 9512 \\
6.918293 \\
\end{array}$ \\
\hline & & .2 & 33 & 3.8 & 7.610122 & & & .6 & 30 & 3.5 & 6.918293 \\
\hline & & .25 & 30 & 4.25 & 6.918293 & & & .7 & 30 & 6.475 & 6.918293 \\
\hline & & 3 & undefined & 63 & undefined & & & 8 & 30 & 6.7 & 6.918293 \\
\hline & & 35 & & & divelined & & & .9 & 30 & 6.125 & 6.918293 \\
\hline & & .25 & undefined & 6.3 & undefined & & & $\frac{1}{15}$ & 30 & 4.8 & $\begin{array}{l}6.918293 \\
6.918293\end{array}$ \\
\hline
\end{tabular}

Table 3: The variation of Position of Plane-Convex lens

Table 4: The vaniation of Hole Radius \& Position of Conerex - Conver lens (Rectanglaser Crystal

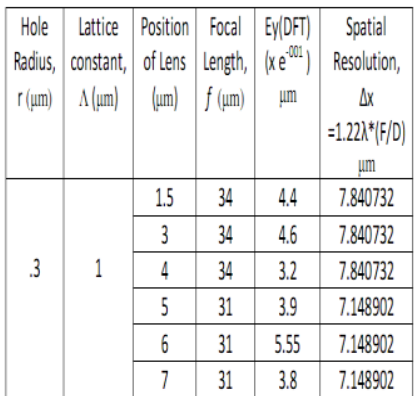

\begin{tabular}{|c|c|c|c|c|}
\hline $\begin{array}{c}\text { Hole Radius, } \\
\text { (um) }\end{array}$ & Distance (um) & $\begin{array}{c}\text { Focal Length, } \\
f(u m)\end{array}$ & $\begin{array}{l}\text { Ey(DFT) } \\
\left(x e^{\text {OWIII }} \mid\right. \\
\mu \mathrm{m}\end{array}$ & $\begin{array}{c}\text { Spatial Resolution, } \\
\Delta x=1.221^{*} \\
(F / 0) \\
\text { min }\end{array}$ \\
\hline \multirow{5}{*}{2} & 4 & 35.5 & 2.775 & 8.186646 \\
\hline & 2 & 35.5 & 3.5 & 8.186646 \\
\hline & 0 & 36 & 5.25 & 8.301951 \\
\hline & 4 & 36.5 & 2.7 & 8.417256 \\
\hline & 4 & 39 & 2.9 & 8.99378 \\
\hline 3 & \multirow{4}{*}{0} & 40 & 3.3 & 9.22439 \\
\hline A & & 31 & 3.6 & 7.148902 \\
\hline 5 & & 28 & 4.75 & 6.457073 \\
\hline 1 & & 38 & 3.4 & 8.763171 \\
\hline
\end{tabular}

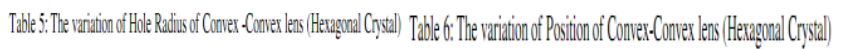

\begin{tabular}{|c|c|c|c|c|c|c|c|c|c|}
\hline $\begin{array}{l}\text { Hole } \\
\text { Radius, }, \\
(m)\end{array}$ & $\begin{array}{c}\text { Distance } \\
(\mathrm{m})\end{array}$ & $\begin{array}{l}\text { Focal } \\
\text { lenghth, } \\
f(\text { ing) }\end{array}$ & 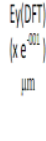 & 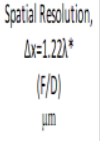 & $\begin{array}{l}\text { Hole } \\
\text { Radius, } 1 \\
\text { (un) }\end{array}$ & $\begin{array}{l}\text { Distance } \\
(\mathrm{min})\end{array}$ & $\begin{array}{c}\text { Focal length, } \\
f(u m)\end{array}$ & 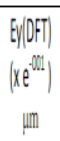 & 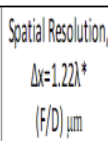 \\
\hline 1 & \multirow{4}{*}{0} & 38 & 3 & 8.763171 & & 0 & 36.45 & 4.75 & 8.405726 \\
\hline .2 & & 39 & 4.625 & 8.99378 & & +2 & Undefined & 2.5 & Undefined \\
\hline$\frac{3}{4}$ & & $\begin{array}{r}36.45 \\
32\end{array}$ & $\begin{array}{r}4.625 \\
34\end{array}$ & 73375512 & & +4 & Undefined & 2 & Undefined \\
\hline .5 & & 31 & 3.8 & 7.149802 & & 2 & 36.45 & 3.5 & 8.405726 \\
\hline
\end{tabular}




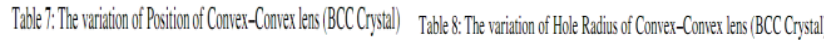

\begin{tabular}{|c|c|c|c|c|c|c|c|c|c|}
\hline \multirow[t]{2}{*}{$\begin{array}{c}\text { Hole } \\
\text { Radius, } \\
(\mathrm{um})\end{array}$} & \multirow[t]{2}{*}{$\begin{array}{l}\text { Distance } \\
(\mathrm{min})\end{array}$} & \multirow[t]{2}{*}{$\begin{array}{l}\text { Focal } \\
\text { length, } \\
f(\text { um) }\end{array}$} & \multirow[t]{2}{*}{ 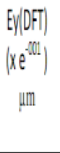 } & \multirow{2}{*}{$\begin{array}{c}\text { Spatiala Resolution, } \\
\Delta x \\
=1.222^{*} \mid[\mid[\mid 0) \\
u m\end{array}$} & $\begin{array}{c}\text { Distance } \\
\text { (um) }\end{array}$ & $\begin{array}{l}\text { Hole } \\
\text { Radius, r } \\
\text { (um) }\end{array}$ & $\begin{array}{l}\text { Focal } \\
\text { Length, } \\
f(\text { (im) }\end{array}$ & $\begin{array}{c}E y(D F) \\
\left(x e^{(01)}\right) \\
\mu m\end{array}$ & $\begin{array}{c}\text { Spatial Resolution, } \\
\Delta x \\
=1.221^{*}(F / D) \\
\mathrm{um}\end{array}$ \\
\hline & & & & & \multirow{4}{*}{0} & -1 & 50 & 3.1 & 8.763171 \\
\hline \multirow{3}{*}{.3} & 0 & 33 & 1.6 & 7.610122 & & .2 & 36.5 & 2.6 & 8.417256 \\
\hline & 2 & 34 & 5 & 7.840732 & & 3 & 33 & 1.6 & 7.610122 \\
\hline & +2 & 34 & 2.6 & 7.840732 & & $\frac{4}{5}$ & & 3.6 & 512 \\
\hline
\end{tabular}

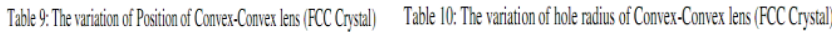

\begin{tabular}{|c|c|c|c|c|c|c|c|c|c|}
\hline $\begin{array}{l}\text { Hole } \\
\text { Radius, } \\
\mathrm{r}(\mathrm{min})\end{array}$ & $\begin{array}{l}\text { Distance } \\
\text { (um) }\end{array}$ & $\begin{array}{l}\text { Focal length, } \\
f(\text { (um) }\end{array}$ & 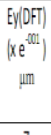 & $\begin{array}{c}\text { Spatia Resolution, } \\
\Delta x \\
=1.221 *(F / D) \\
\mu \mathrm{m}\end{array}$ & $\begin{array}{l}\text { Distance } \\
\text { (um) }\end{array}$ & $\begin{array}{l}\text { Hole } \\
\text { Radius, } \\
\mathrm{r}(\mathrm{m})\end{array}$ & $\begin{array}{l}\text { Focal } \\
\text { Length, } \\
f(\mathrm{~min})\end{array}$ & $\begin{array}{c}\text { Ey(DFF) } \\
\left(x \mathrm{e}^{\mathrm{em} / 1}\right) \\
\mu \mathrm{m}\end{array}$ & $\begin{array}{c}\text { Spatiala Resolution } \\
\Delta x \\
=1.222^{*}|F|(\mid) \mid \\
\mu m \\
m\end{array}$ \\
\hline \multirow{5}{*}{3} & 0 & undefined & 7 & undefined & \multirow{5}{*}{0} & .1 & 36.45 & 4.2 & 8.405726 \\
\hline & 2 & 30 & 6.8 & 6.918293 & & .2 & 34 & 2.8 & 7.840732 \\
\hline & +2 & undefined & 7.3 & undefined & & .3 & 30 & 7.4 & 6.918293 \\
\hline & +4 & undefined & 4.9 & undefined & & .4 & 31 & 7.3 & 7.148902 \\
\hline & 4 & 30 & 5.8 & 6.918293 & & .5 & 30 & 7 & 6.918293 \\
\hline
\end{tabular}

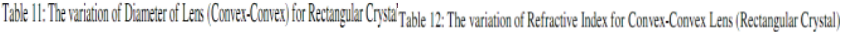

\begin{tabular}{|c|c|c|c|c|c|c|c|c|c|}
\hline \multirow[t]{2}{*}{$\begin{array}{l}\begin{array}{l}\text { Distance } \\
\text { (inf) }\end{array}\end{array}$} & \multirow[t]{2}{*}{$\begin{array}{l}\text { Hole } \\
\text { Padilis, } \\
\mathrm{r}(\mathrm{m} \text { (I) }\end{array}$} & \multirow[t]{2}{*}{\begin{tabular}{|c|} 
Varition of \\
Diameter \\
of thelens \\
$D(\mu n)$
\end{tabular}} & \multirow[t]{2}{*}{$\begin{array}{l}\text { Foral } \\
\text { Lenghth, } \\
\text { f(ur) }\end{array}$} & \multirow[t]{2}{*}{ 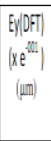 } & \multirow[t]{2}{*}{ 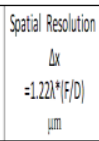 } & $\begin{array}{l}\text { Refractive } \\
\text { Indoer( } \mathrm{Rl})\end{array}$ & $\begin{array}{l}\text { Focel length, } \\
f(\mathrm{~mm})\end{array}$ & 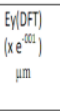 & \multirow[t]{2}{*}{ 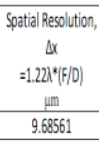 } \\
\hline & & & & & & 1 & 42 & 2.2 & \\
\hline \multirow{5}{*}{0} & \multirow{5}{*}{3} & 8.2 & 34 & 1.8 & 7.80732 & 1.3 & 36.45 & 3 & 8.4057276 \\
\hline & & 10.25 & 24.5 & 3.5 & 4559551 & 1.4505 & 36.45 & 2.6 & 8.405726 \\
\hline & & 123 & 30 & 2.4 & 46612195 & 1.5 & 36.45 & 1.9 & 8.405726 \\
\hline & & 164 & 36.65 & 19 & & 2 & 36.45 & 2.3 & 8.4057276 \\
\hline & & & & & 4.20180805 & 3 & Undefined & 7.3 & Undefined \\
\hline
\end{tabular}

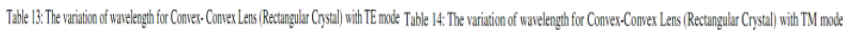

\begin{tabular}{|c|c|c|c|c|c|c|c|}
\hline \multicolumn{4}{|c|}{ TEmode } & \multicolumn{4}{|c|}{ TM mode } \\
\hline $\begin{array}{c}\text { Warelength, } \\
\lambda(\text { (min) }\end{array}$ & $\begin{array}{c}\text { focol length, } \\
f(\mathrm{~min})\end{array}$ & 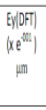 & 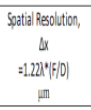 & $\begin{array}{c}\text { Wavelength, } \\
\lambda(\mu m)\end{array}$ & $\begin{array}{c}\text { Focal Length, } \\
f(\mathrm{~mm})\end{array}$ & 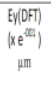 & $\begin{array}{c}\text { Spatial Resolution, } \\
\Delta x \\
=1.22 x^{*}(F / D) \\
\mu m\end{array}$ \\
\hline 0.85 & Undefined & 1.75 & Undefined & 0.85 & 40 & 54 & 9.22439 \\
\hline 1.00 & Undefined & 5.6 & Undefined & 1.00 & 40 & 127 & 9.22239 \\
\hline 1.20 & Undefned & 3.9 & Undefined & 1.20 & 36.45 & 94 & 8.405726 \\
\hline 1.35 & 36.45 & 3.6 & 8.45726 & 1.35 & 36.45 & 35 & 8.405726 \\
\hline 1.45 & 36.15 & 3.4 & 8.467726 & 1.45 & 36 & 96 & 8.301951 \\
\hline 1.55 & 36.5 & 2.6 & 8405726 & 1.55 & 36 & 117 & 8301951 \\
\hline 1.65 & 36.15 & 3.4 & 8445726 & 1.65 & 36 & 107 & 8301951 \\
\hline 1.75 & 36.15 & 24 & 8445726 & 1.75 & 36.45 & 24 & 8.405726 \\
\hline
\end{tabular}

Table 15: Optimization of parameters for crystals (Rectangular Crystal)

\begin{tabular}{|c|c|c|c|c|c|}
\hline $\begin{array}{l}\text { Crystal } \\
\text { Types }\end{array}$ & $\begin{array}{c}\text { Optimized } \\
\text { Lattice } \\
\text { constant, } \\
\Lambda(\mu \mathrm{m})\end{array}$ & $\begin{array}{c}\text { Optimized } \\
\text { Hole } \\
\text { Radius, r } \\
(\mu \mathrm{m})\end{array}$ & $\begin{array}{l}\text { Focal } \\
\text { Length, } \\
f(\mu \mathrm{m})\end{array}$ & $\begin{array}{c}\text { Ey(DFT) } \\
\left(\mathrm{xe}^{-0 \mathrm{CO1}}\right) \\
\mu \mathrm{m}\end{array}$ & $\begin{array}{c}\text { Spatial } \\
\text { Resolution, } \\
\Delta x \\
=1.22 \lambda^{*}(\mathrm{~F} / \mathrm{D} \\
\mathrm{um}\end{array}$ \\
\hline Rectangular & & 0.3 & 40 & 3.3 & 9.22439 \\
\hline Hexagonal & & 0.4 & 32 & 3.4 & 7.379512 \\
\hline$B C C$ & & 0.3 & 34 & 5 & 7.840732 \\
\hline $\mathrm{FCC}$ & & 0.2 & 34 & 2.8 & 7.840732 \\
\hline
\end{tabular}

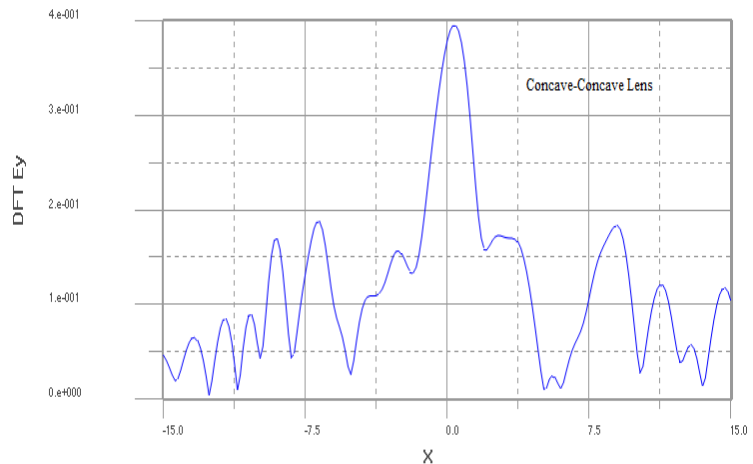

(A4)

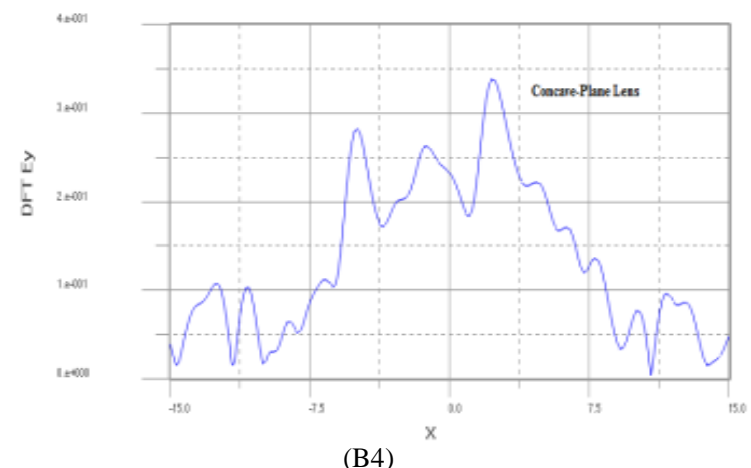

(B4)

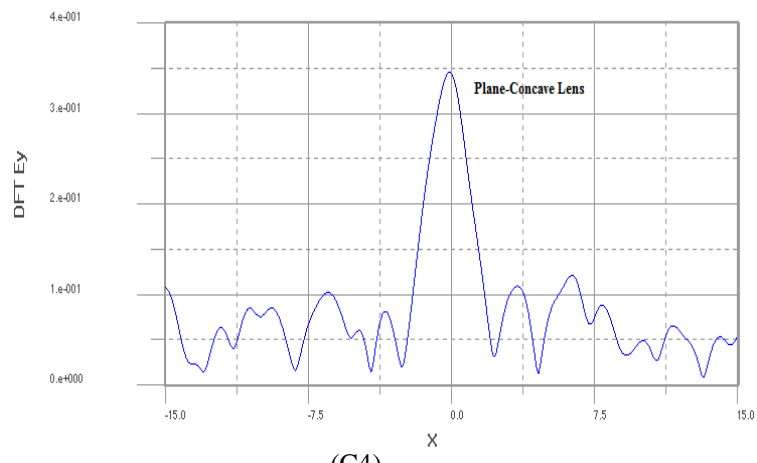

(C4) 


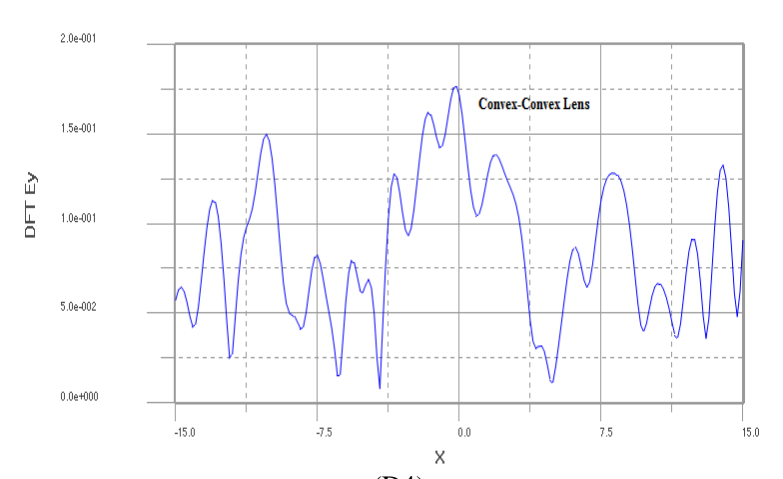

(D4)

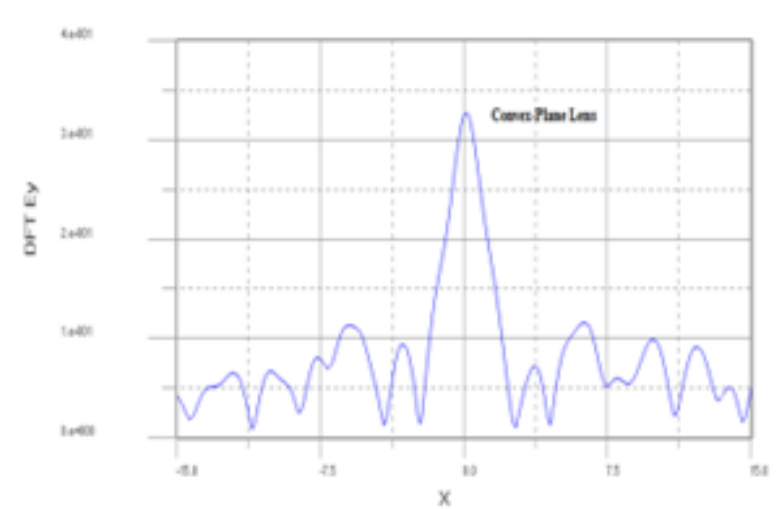

(E4)

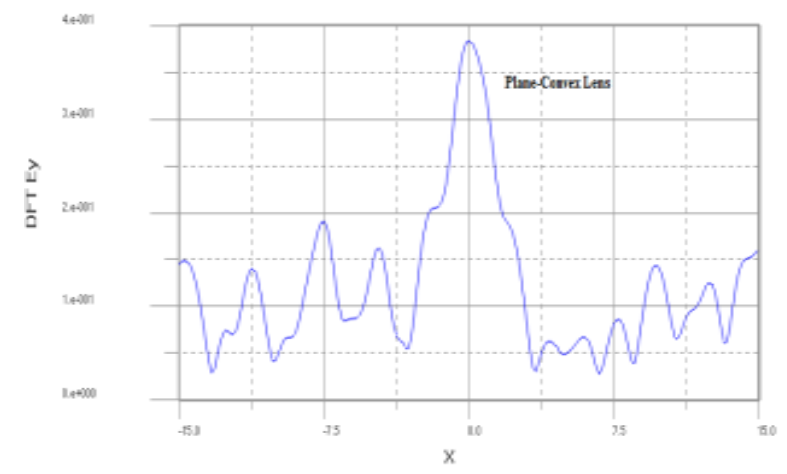

(F4)

Figure 4. DFT Ey curves for Concave-Concave(A4), Concave-Plane(B4), Plane-Concave(C4), Convex-Convex (D4), Convex-Plane(E4), Plane-Covex Lens(F4)

\section{CONCLUSION}

An ideal case is considered without any temperature effect but temperature will cause the change of the reflective index and consequently to focal length $(f)$ and the spatial resolution $(\Delta \mathrm{x})$ for TM Mode. For TE Mode, it will remain unaffected. Moreover, unlike optical lenses- Sub-wavelength focusing has been found for photonic lenses but axis dependence and change of the focal length $(f)$ with diameter is almost same. With optimize parameters: Hole radiuses (r), $0.2 \mu \mathrm{m}$ (FCC),
$0.3 \mu \mathrm{m}$ (BCC, Rectangular), $0.4 \mu \mathrm{m}$ (Hexagonal), lattice constant, $\Lambda=1$, distances or position of lenses is zero $\mu \mathrm{m}$, reflective index $\mathrm{n}=1.4505$, focal length $(f): 40 \mu \mathrm{m}, 32 \mu \mathrm{m}, 34 \mu \mathrm{m}$, $34 \mu \mathrm{m}$ and spatial resolution $(\Delta \mathrm{x}): 9.22 \mu \mathrm{m}, 7.37 \mu \mathrm{m}, 7.84 \mu \mathrm{m}$, $7.84 \mu \mathrm{m}$ (for Rectangular, Hexagonal, BCC, FCC). Finally, with optimized parameters, Concave-Concave photonic lens is found as optimal photonic lens for Rectangular crystal structure that shows.

\section{ACKNOWLEDGEMENT}

We would like to thanks all reviewers for their valuable comments. All thanks due especially to my parents for their prayer and inspiration, which has helped me in becoming what I am today.

\section{REFERENCES}

[1] J. D. Joannopoulos, R. D. Meade, and J. N. Winn "Photonic Crystals: Molding the Flow of Light", Princeton University Press (1999).

[2] Qi Wu, John M. Gibbons and Wounjhang Park, "Graded Negative Index Lens by Photonic Crystal", Optics Express, Vol.16, No. 21, 1694216949, (2008).

[3] A. Taflove,"Compuatational Electrodynamics: The Finite-Difference Time-Domain Method", Artech House, Boston, MA (1995).

[4] N. Palka, W. Ciurapinski and J. Wróbel, "Focusing with 2D Square Photonic Crystal with Concavo-Concavo Boundaries", Optical and Acoustical Methods in Science and Technology, Vol.116, No. 3, 368370, (2009).

[5] I. V. Minin, O. V. Minin, Y. R. Triandaphilov and V. V. Kotlyar, "Subwavelength Diffractive Photonic Crystal Lens", progress in Electromegneties Research B, Vol. 7, 257-264, (2008).

[6] Hamza Kurt, "Graded index photonic crystals", Optics Express, Vol.15, No. 3, 1241-1253, (2007).

[7] Zhaolin Lu, Shouyuan Shi, Christopher A. Schuetz, Janusz A. Murakowski, and Dennis W. Prather, "Three-dimensional photonic crystal flat lens by full 3D negative refraction", Optics Express, Vol.13, No. 15, 5592-5599, (2005).

[8] X. Wang, Z. F. Ren and K. Kempa, "Unrestricted superlensing in a triangular two-dimensional photonic crystal”, Optics Express, Vol.12, No. 13, 2919-2924, (2004).

\section{AUTHORS PROFILE}

Rajib Ahmmed was born in Kishoregonj, Bangladesh on 05 May, 1986. He received the B. Sc. Honours and M.Sc degree in Applied Phsics, Electronics and Communication Engineering from Dhaka University, Dhaka, Bangladesh, in 2008. He is a Lecturer (study leave) of University of Information Technology \& Sciences (UITS), Baridhara, Dhaka, Bangladesh. Now, he doing Erasmus Mundus MASters on Photonic NETworks engineering (MAPNET), Institute of Scuola Superiore Sant' Anna, Pisa, Italy and Phone: +393892320071. His research interest concerns on the photonic related works.

Mahidul Haque Prodhan is with the Applied physics, Electronics \& Communication Engineering, Institute of University of Dhaka, Dhaka1000, Bangladesh;

Rifat Ahmmed is with the Electronics and Telecommunication Engineering department, Institute of Rajshahi University of engineering and Technology, Rajshahi-6204, Bangladesh, Phone: +880-1914961164; 